\title{
Categorization of Third-Party Apps in Electronic Health Record App Marketplaces: Systematic Search and Analysis
}

Jordon Ritchie ${ }^{*}$, BSc; Brandon Welch ${ }^{*}, \mathrm{MSc}, \mathrm{PhD}$

Medical University of South Carolina, Charleston, SC, United States

*all authors contributed equally

\section{Corresponding Author:}

Brandon Welch, MSc, PhD

Medical University of South Carolina

135 Cannon Street, MSC 200

Suite 405

Charleston, SC, 29425

United States

Phone: 18435183769

Email: welchbm@musc.edu

\section{Abstract}

Background: Third-party electronic health record (EHR) apps allow health care organizations to extend the capabilities and features of their EHR system. Given the widespread utilization of EHRs and the emergence of third-party apps in EHR marketplaces, it has become necessary to conduct a systematic review and analysis of apps in EHR app marketplaces.

Objective: The goal of this review is to organize, categorize, and characterize the availability of third-party apps in EHR marketplaces.

Methods: Two informaticists (authors JR and BW) used grounded theory principles to review and categorize EHR apps listed in top EHR vendors' public-facing marketplaces.

Results: We categorized a total of 471 EHR apps into a taxonomy consisting of 3 primary categories, 15 secondary categories, and 55 tertiary categories. The three primary categories were administrative $(\mathrm{n}=203,43.1 \%)$, provider support $(\mathrm{n}=159,33.8 \%)$, and patient care $(\mathrm{n}=109,23.1 \%)$. Within administrative apps, we split the apps into four secondary categories: front office $(\mathrm{n}=77$, $37.9 \%)$, financial $(n=53,26.1 \%)$, office administration $(n=49,24.1 \%)$, and office device integration $(n=17,8.4 \%)$. Within the provider support primary classification, we split the apps into eight secondary categories: documentation $(\mathrm{n}=34,21.3 \%)$, records management $(\mathrm{n}=27,17.0 \%)$, care coordination $(\mathrm{n}=23,14.4 \%)$, population health $(\mathrm{n}=18,11.3 \%)$, EHR efficiency $(\mathrm{n}=16,10.1 \%)$, ordering and prescribing $(n=15,9.4 \%)$, medical device integration $(n=13,8.2 \%)$, and specialty EHR $(n=12,7.5 \%)$. Within the patient care primary classification, we split the apps into three secondary categories: patient engagement $(\mathrm{n}=50,45.9 \%)$, clinical decision support $(n=40,36.7 \%)$, and remote care $(n=18,16.5 \%)$. Total app counts varied substantially across EHR vendors. Overall, the distribution of apps across primary categories were relatively similar, with a few exceptions.

Conclusions: We characterized and organized a diverse and rich set of third-party EHR apps. This work provides an important reference for developers, researchers, and EHR customers to more easily search, review, and compare apps in EHR app marketplaces.

(JMIR Med Inform 2020;8(5):e16980) doi: 10.2196/16980

\section{KEYWORDS}

electronic health records; medical informatics; software; interoperability; apps; app marketplace

\section{Introduction}

The electronic health record (EHR) stores patient health information, automates clinical workflows, and supports other care-related functions such as clinical decision support [1]. Clinical and governmental drivers have facilitated widespread adoption of EHRs in health care worldwide [2]. Health care providers rely on EHRs to perform essential functions such as documenting patient encounters, providing clinical decision support, and engaging patients in their own care [3,4]. However, EHR implementation hurdles, usability flaws, and poor interoperability, among other issues, keep EHRs from delivering 
their full potential benefit to health care organizations [5]. The variation in EHR implementation across health organizations contributes to these problems as each organization may rely on different methods to integrate additional value into their EHR systems. Some organizations may leverage custom integration with third-party applications whereas others may resort to in-house development or other integration strategies to support their needs [6,7]. In any case these integrations tend to be time-consuming, expensive, and limited for use only within their respective organizations [8]. Ideally, a successful information technology (IT) application that integrates with an EHR at one organization would be available for the same integration with an EHR at another organization, regardless of EHR vendor [9].

The EHR app model, inspired by smartphone app marketplaces, has been proposed to increase flexibility and availability of EHR integrations while also fostering innovation in health IT [9-11]. This approach is made possible by increased EHR interoperability and standardized access to EHR data through application programming interfaces (APIs) and standards such as FHIR (Fast Healthcare Interoperability Resources) [12]. One early implementation, the SMART (Substitutable Medical Applications, Reusable Technologies) App Gallery [13] is an example of an EHR app platform that heavily leverages the FHIR standard to enable a plug-and-play style of integration with participating EHRs [14]. SMART app development depends heavily on two major concepts - apps must be both substitutable and reusable. A substitutable app accesses EHR data and can be easily added, replaced, or deleted within an EHR. This allows health care organizations to choose the app that best fits their needs [15]. A reusable app is developed once but can be installed by many clients potentially across multiple EHRs [15]. The FHIR standard serves as the common data specification that both EHR vendor APIs and SMART APIs adhere to in order to interoperate. An EHR app platform built on these concepts increases access to health IT solutions for health care organizations and allows third-party app developers to compete in a market driven by the value and price of their app [9]. Motivated by these benefits [16], major EHRs have started to create their own app marketplaces to encourage development of apps on their own platforms [15]. There are now hundreds of apps available on EHR app marketplaces.

Given the widespread utilization of EHRs and the emergence of EHR app marketplaces, it has become necessary to conduct a systematic search and analysis of apps in EHR app marketplaces in an effort to help organize, categorize, and characterize available EHR apps. This study will help health professionals, researchers, and developers understand the currently available technologies; make it easier to review, search, and compare available EHR apps; provide a common vocabulary to facilitate communication; identify where gaps and opportunities exist for research and development; and justify investment into the research and development of new EHR apps.

\section{Methods}

\section{App Extraction}

We identified and reviewed all known apps in public-facing marketplaces of the top 10 EHR vendors in the United States, which include (in order of market share) Epic Systems Corporation; Allscripts; eClinicalWorks, LLC; NextGen Healthcare; GE Healthcare; athenahealth, Inc; Cerner Corporation; Greenway Health LLC; Practice Fusion; and eMDs [17]. GE Healthcare did not have a public facing app marketplace at the time of this writing and Practice Fusion was recently acquired by Allscripts [18]. The leading vendors in the US market were chosen for analysis because they had publicly available app marketplaces (with the exception of GE Healthcare), and they represented a cluster of vendors serving the majority of a common set of customers.

We used custom web scrapers and public ReST (Representational State Transfer) endpoints from these EHR marketplaces to gather EHR app data such as name, description, links, website, EHR-defined app categories, ratings, reviews, EHR versions, and other available information. This information was recorded in our EHR app database wherein we consolidated duplicate EHR apps that were listed in multiple EHR marketplaces. The last data extraction occurred in February 2019. Clear indication of FHIR compatibility was not consistently present in the extracted data within or across EHR marketplaces. Marketplace offerings that offered professional services without clear evidence of EHR integration (eg, website builders or marketing services), teams of professionals granted access to EHR interfaces (eg, offsite medical coders), and medical devices without EHR integration (eg, stand-alone weight scale) were not considered apps and were excluded.

\section{App Review}

Two informaticists (authors JR and BW) used grounded theory principles to create categories that emerged from the EHR app information [19]. EHR app classifications were created inductively by each reviewer independently based upon available information about each EHR app. Importantly, not all data fields were available across all EHRs. For example, not all marketplaces included information indicating whether the app was open source or a commercial offering. Even though some marketplaces included this information, apps were generally classified based on the information available that was common across apps from all marketplaces. When the EHR app information was either inadequate or missing, making it difficult to accurately classify the app, we referenced the app developer's website. As common EHR app features, functions, or purposes emerged, we created categories to group similar apps. Where similarity between categories existed, they were related to form larger, more inclusive categories. Conversely, if sufficient EHR app divergence existed within a category, we subclassified the apps into more unified categories [20]. We drew category names from app descriptions, EHR-designated classifications, and common industry concepts. A minimum of three apps were required to form a category.

Following the review and initial classification by each reviewer independently, a joint review process commenced between the 
two reviewers. The reviewers worked together to come to a consensus on category names, the organization of the taxonomic class hierarchy, and the correct classification of each EHR app. To facilitate the joint review process, a database with all EHR app information as well as notes from each reviewer and their initial classifications were utilized. Each EHR app was reviewed and discussed together. As consensus categories emerged, formal definitions for each category were created and refined. A final classification was used to denote the consensus classification. Consensus was reached when both reviewers agreed with the classification. In cases where the reviewers failed to reach consensus, a third-party arbitrator was available. Through multiple rounds of discussion and debate, a consensus EHR app taxonomy emerged.

\section{Results}

\section{App Extraction}

Of the eight EHRs with public-facing marketplaces, we identified a total of 749 offerings. The total number of offerings for marketplaces ranged from 21 (eMD) to 227 (Athenahealth).
In total, 153 offerings were listed on at least two EHR marketplaces, resulting in a total of 596 unique offerings; 125 were excluded from consideration for not meeting our inclusion criteria, which resulted in 471 unique apps being incorporated into our taxonomy. We categorized the EHR app into a taxonomy consisting of 3 primary categories, 15 secondary categories, and 55 tertiary categories. The three primary categories were administrative ( $\mathrm{n}=203$ apps, $43.1 \%$ ), provider support $(n=159,33.8 \%)$, and patient care $(n=109,23.1 \%)$.

For each EHR, the distribution of apps across the primary categories followed a similar trend. In general, administrative apps make up the greatest portion of EHR apps, followed by provider support apps and then primary care apps. Interestingly, Cerner's marketplace has a higher ratio of patient care apps, followed by provider support and then administrative. There was also a large variability in the number of listings excluded for not meeting criteria for being an app between EHR marketplaces, with eClinicalWorks accounting for 65 of 125 $(52 \%)$ excluded offerings (Table 1$)$. Each primary category is described in further detail below.

Table 1. Distribution of app marketplace offerings across primary categories by electronic health record (EHR) vendors.

\begin{tabular}{|c|c|c|c|c|}
\hline \multirow[t]{2}{*}{ EHR vendor } & \multicolumn{4}{|l|}{ Primary category } \\
\hline & Administrative, $\mathrm{n}(\%)$ & Provider support, n (\%) & Patient care, n (\%) & Not an app, n (\%) \\
\hline Athenahealth $(n=222)$ & $90(40.5)$ & $63(28.4)$ & $45(20.3)$ & $24(10.8)$ \\
\hline eClinicalWorks $(n=116)$ & $23(19.8)$ & $14(12.1)$ & $14(12.1)$ & $65(56.0)$ \\
\hline Epic $(n=113)$ & $46(40.7)$ & $33(29.2)$ & $30(26.6)$ & $4(3.5)$ \\
\hline Allscripts ( $\mathrm{n}=110)$ & $33(30.0)$ & $47(42.7)$ & $28(25.5)$ & $2(1.8)$ \\
\hline Greenway $(\mathrm{n}=87)$ & $48(55.2)$ & $19(21.8)$ & $4(4.6)$ & $16(18.4)$ \\
\hline Nextgen $(n=37)$ & $10(27.0)$ & $14(37.8)$ & $4(10.8)$ & $9(24.3)$ \\
\hline Cerner $(n=28)$ & $1(3.6)$ & $10(35.7)$ & $17(60.7)$ & $0(0.0)$ \\
\hline $\operatorname{eMD}(n=21)$ & $9(42.9)$ & $3(14.3)$ & $0(0.0)$ & $9(42.9)$ \\
\hline
\end{tabular}

\section{App Review}

\section{Administrative}

The 203 administrative apps facilitate the administrative functions of a hospital or clinic. Within this classification, we split the apps into four secondary categories: front office $(\mathrm{n}=77$, $37.9 \%)$, financial $(n=53,26.1 \%)$, office administration $(n=49$, $24.1 \%)$, and office device integration $(n=17,8.4 \%)$. The administrative app categories, descriptions, and counts are listed in Table 2. 
Table 2. Category definitions and counts for administrative apps $(n=203)$.

\begin{tabular}{|c|c|c|}
\hline Categories & Category descriptions & Count, $\mathrm{n}(\%)$ \\
\hline Administrative & Facilitates the conduct of administrative functions of a hospital or clinic setting & $203(100.0)$ \\
\hline Front office & Helps support front office staff interaction with patients & $77(37.9)$ \\
\hline Scheduling & Helps schedule and manage patient appointments & $32(15.7)$ \\
\hline Patient check-in & Helps manage the patient check-in process & $12(5.9)$ \\
\hline Patient communication & Facilitates communication with patient for administrative purposes & $12(5.9)$ \\
\hline Document management & Helps capture and manage documents & $10(4.9)$ \\
\hline Answering service & Captures information related to after-hours patient calls & $4(2.0)$ \\
\hline Phone triage & Facilitates triage according to industry standard protocols & $3(1.5)$ \\
\hline Financial & Helps manage the financial needs of the clinic & $53(26.1)$ \\
\hline Patient billing & Captures and processes payment information from patients & $20(9.9)$ \\
\hline Insurance & Facilitates claims and authorization & $13(6.4)$ \\
\hline Collections & Manages patient collections & $8(3.9)$ \\
\hline Medical coding & Improves accuracy and efficiency of medical coding & $7(3.4)$ \\
\hline Patient pay estimation & Estimates cost of care & $3(1.5)$ \\
\hline Office administration & Supports the administrative needs of the clinic & $49(24.1)$ \\
\hline Analytics and reporting & Helps track, analyze, and report on clinical operations & $17(8.4)$ \\
\hline Patient experience & Measures the clinical experience of the patient & $13(6.4)$ \\
\hline Inventory management & Tracks inventory of medical products & $7(3.4)$ \\
\hline $\mathrm{IT}^{\mathrm{a}}$ systems management & Supports the IT system needs of a health care organization & $7(3.4)$ \\
\hline Compliance & Helps maintain, track, and/or report compliance & $4(2.0)$ \\
\hline Office device integration & Device used by office staff for administrative purposes & $17(8.4)$ \\
\hline Scanner integration & Integrates scanners with the $\mathrm{EHR}^{\mathrm{b}}$ & $7(3.4)$ \\
\hline Printer integration & Integrates printers with the EHR & $3(1.5)$ \\
\hline Signature pad integration & Integrates a signature pad with the EHR & $3(1.5)$ \\
\hline
\end{tabular}

${ }^{\mathrm{a}} \mathrm{IT}$ : information technology.

${ }^{\mathrm{b}}$ EHR: electronic health record.

\section{Provider Support}

We identified 159 provider support apps, which we defined as apps that primarily support the functions of care providers in their delivery of health care to patients. Within the provider support primary classification, we split the apps into eight secondary categories: documentation $(n=34,21.3 \%)$, records management $(n=27,17.0 \%)$, care coordination $(n=23,14.4 \%)$, population health $(n=18,11.3 \%)$, EHR efficiency $(n=16,10.1 \%)$, ordering and prescribing $(\mathrm{n}=15,9.4 \%)$, medical device integration $(n=13,8.2 \%)$ and specialty EHR $(n=12,7.5 \%)$. The provider support app categories, descriptions, and counts are in Table 3. 
Table 3. Category definitions and counts for provider support apps $(n=159)$.

\begin{tabular}{|c|c|c|}
\hline Categories & Category descriptions & Count, $\mathrm{n}(\%)$ \\
\hline Provider support & Supports the functions of care providers in their delivery of health care & $159(100.0)$ \\
\hline Documentation & Facilitates the collection and management of patient information & $34(21.3)$ \\
\hline Dictation and transcription & Transcribes dictated clinical narratives into clinical notes & $12(7.5)$ \\
\hline Structured documentation & Facilitates efficient and accurate documentation & $7(4.4)$ \\
\hline Image capture & Captures images for documentation (usually a mobile device) & $6(3.8)$ \\
\hline Natural language processing & Uses natural language processing to process unstructured data & $6(3.8)$ \\
\hline Records management & Supports access to or management of records & $27(17.0)$ \\
\hline Image management & Allows access to or management of images, including RIS/PACS ${ }^{\mathrm{a}}$ systems & $11(6.9)$ \\
\hline Legacy/migration & $\begin{array}{l}\text { Provides access to legacy medical records or facilitates the conversion of paper } \\
\text { records to electronic records }\end{array}$ & $8(5.0)$ \\
\hline Access & Consolidates patient records in one view or allows access via mobile device & $5(3.1)$ \\
\hline Backup & Saves data in an alternate form that can be accessed in the event of an outage & $3(1.9)$ \\
\hline Care coordination & Helps care team members coordinate their care for a patient & $23(14.4)$ \\
\hline Clinic scheduling & Manages the scheduling and workflow of providers in a clinic & $7(4.4)$ \\
\hline Service directory & Provides a list of services or providers to refer or access & $7(4.4)$ \\
\hline Provider communication & Facilitates the communication between care team members about a patient & $6(3.8)$ \\
\hline Population health & Helps manage that health of a population or group of patients & $18(11.3)$ \\
\hline Chronic care management & Helps providers manage chronic conditions in patients & $10(6.3)$ \\
\hline Annual wellness visit & Facilitates annual wellness visit scheduling and reporting & $4(2.5)$ \\
\hline Population risk assessment & Helps identify and manage at-risk patients & $4(2.5)$ \\
\hline $\mathbf{E H R}^{\mathrm{b}}$ efficiency & Makes the EHR easier or more efficient for the provider to use & $16(10.1)$ \\
\hline Information display & Consolidates patient record into easily consumed dashboards, reports, and infographics & $9(5.7)$ \\
\hline Ordering and prescribing & Facilitates the ordering or prescribing of a device, substance, or service & $15(9.4)$ \\
\hline $\begin{array}{l}\text { Prescription drug monitoring } \\
\text { program }\end{array}$ & Provide access to state Prescription Drug Monitoring Program databases & $5(3.1)$ \\
\hline Pharmacy & Manages electronic prescription renewal and ordering & $4(2.5)$ \\
\hline Medical equipment & Manages electronic ordering of DME ${ }^{\mathrm{c}}$ & $3(1.9)$ \\
\hline Image ordering & Facilitates image ordering or helps manage image ordering workflow & $3(1.9)$ \\
\hline Medical device integration & Device used by health care provider for clinical purposes & $13(8.2)$ \\
\hline Cardiac devices & Collects data from cardiac devices & $5(3.1)$ \\
\hline Digital scales & Collects data from digital scales & $3(1.9)$ \\
\hline Specialty EHR & Extends the functions of an EHR to support a specific clinical domain or specialty & $12(7.5)$ \\
\hline Obstetrics & Extends the EHR to provide functionality for prenatal and perinatal data management & $3(1.9)$ \\
\hline Anesthesia & Extends the EHR to provide functionality for Anesthesia data management & $3(1.9)$ \\
\hline
\end{tabular}

${ }^{a}$ RIS/PACS: Radiology Information System/Picture Archiving and Communication System.

${ }^{\mathrm{b}}$ EHR: electronic health record.

${ }^{\mathrm{c}}$ DME: durable medical equipment.

\section{Patient Care}

The 109 patient care apps we identified facilitate the provision of clinical care between a health care provider and a patient. Within the patient care primary classification, we split the apps into three secondary categories: patient engagement $(n=50$, $45.9 \%)$, clinical decision support $(n=40,36.7 \%)$, and remote care $(n=18,16.5 \%)$. The patient care app categories, descriptions, and counts are listed in Table 4. 
Table 4. Category definitions and counts for patient care apps $(n=109)$.

\begin{tabular}{|c|c|c|}
\hline Categories & Category descriptions & Count, $\mathrm{n}(\%)$ \\
\hline Patient care & Facilitates the provision of clinical care between a health care provider and a patient & $109(100.0)$ \\
\hline Patient engagement & Engages patients in their own health care & 50 (45.9) \\
\hline Patient assessment & Collects patient-reported information for a clinical purposes & $15(13.8)$ \\
\hline Care plan management & $\begin{array}{l}\text { Helps patient follow a provider's rehab instructions, medication schedule, and/or care } \\
\text { plan regimen }\end{array}$ & $10(9.2)$ \\
\hline Patient education & Provides education and instruction resources to patients specific to their care & $9(8.3)$ \\
\hline Health record access & $\begin{array}{l}\text { Allows patients to access, download, and share their medical records, or allows providers } \\
\text { to fulfill medical record requests }\end{array}$ & $6(5.5)$ \\
\hline Patient wearables & Records information from patient wearables (passive involvement) & $6(5.5)$ \\
\hline Clinical decision support & Provides or delivers decision support to providers based on patient data & $40(36.7)$ \\
\hline Ordering $\mathrm{CDS}^{\mathrm{a}}$ & Supports the appropriateness of medication, imaging, and lab test orders & $10(9.2)$ \\
\hline Medication CDS & Provides decision support for medication dosing and monitoring & $9(8.3)$ \\
\hline Patient risk assessment & Assess a patient's health risk & $5(4.6)$ \\
\hline Knowledge management & Provides access to and manages medical knowledge for providers & $5(4.6)$ \\
\hline Patient monitoring & Monitors health of patient and alerts provider of notable changes & $4(3.7)$ \\
\hline Remote care & Supports the provision of care to patient remotely & $18(16.5)$ \\
\hline Telehealth platform & Gives a provider technical capabilities to meet with a patient remotely & $12(11.0)$ \\
\hline Remote consult & Provides access to care providers or specialists who are remote & $5(4.6)$ \\
\hline
\end{tabular}

${ }^{\mathrm{a}} \mathrm{CDS}$ : clinical decision support.

\section{Discussion}

\section{Principal Results}

We conducted a systematic search and analysis of apps in EHR app marketplaces to help organize, categorize, and characterize available EHR apps. This study brings value to the health IT industry because it creates a common vocabulary that can be used to communicate about EHR apps, helps health care organizations identify EHR app solutions, and justifies investment into the research and development of new EHR apps. With this study, we can identify common patterns of EHR integration approaches and create a template to streamline future EHR app development and integration. This helps researchers identify gaps in integration capabilities, standards, or functionalities that need to be addressed by governing bodies, standards organizations, EHR vendors, or EHR app developers.

Our EHR app review organized and characterized 471 unique EHR apps into 3 primary categories, 15 secondary categories, and 55 tertiary categories. Several categories were larger or more well-defined than others. Administrative apps represented the largest share of EHR apps with 203 apps. Provider support and patient care apps were the other primary categories with 159 and 109 EHR apps, respectively. EHR marketplaces tended to reflect this overall trend with the majority of apps falling under the administrative category, followed by provider support and patient care. Cerner followed a distinctly different trend with patient care representing the majority of their apps and only a single administrative app. This may in part be attributed to how Cerner validates apps that are submitted by third-party developers. While all app galleries reviewed here have a submission process, and several list disclaimers that not all submissions may be listed upon submission, Cerner has an additional validation step. Apps that don't meet a certain standard set by Cerner may be rejected or asked to resubmit after outstanding issues are resolved. This adds extra rigor in the Cerner app submission process that may account for the lower total app count in their gallery as well as the different ratio of primary categories observed. The fact that zero offerings in Cerner's gallery were considered "Not an app" and excluded from consideration in our search and analysis may be attributed to their unique validation approach and, generally speaking, Cerner's apps required less attention when assigning apps to categories. However, Cerner also had fewer total offerings listed in their gallery $(n=28)$ than all other vendors except for eMD $(n=21)$. App quality is an important issue and while the right amount of validation is difficult to quantify [8], it is important to note that if validation is too strict, it could suppress innovation and defeat a key purpose of the app model, which allows competition among developers based on app value and price [9]. This allows clients to validate app offerings and reward innovation and the value the apps provide [15].

Interestingly, provider support apps had the greatest variability and ambiguity among the three primary categories of apps. Provider support had more secondary categories than the other two primary categories combined, accounting for 8 of the 15 (53\%) total secondary categories. Many apps offered multiple functionalities or had feature sets that made it difficult to assign secondary and tertiary categories. The value these apps provided and how they were intended to be used by the provider was often unclear. Provider support accounted for 63\% (14/22) of 
apps that were not specific enough to place in a secondary category. Additionally, provider support accounted for $46 \%$ $(25 / 54)$ of apps that were not specific enough to place in a tertiary category. The secondary categories under provider support with the most apps without tertiary categories were specialty $\operatorname{EHR}(6 / 12,50 \%)$, EHR efficiency (7/16, 44\%), and medical device integration $(7 / 13,39 \%)$. Among all other secondary categories, office device integration had the highest percentage of apps that did not fall into a tertiary category (4/17, $24 \%)$, followed by clinical decision support $(7 / 40,18 \%)$. This suggests that the provider support category has the greatest need for further refinement and innovation out of the three primary categories.

The app model is a remedy to the one-size-fits-all strategy that is failing to meet the needs of patients, providers, and administrators in a rapidly evolving landscape [8]. For the app model to be effective, the apps listed need to solve a clearly defined problem instead of offering diverse sets of features and functionalities that begin to approximate a one-size-fits-all-solution. In other words, it needs to be obvious what category the app belongs in. The taxonomy of apps we have curated will help health IT companies and app developers match app development to a well-defined purpose and assist health professionals in identifying gaps in the current set of app categories. As app functionality and feature sets become more cohesive and achieve alignment with specific EHR app categories, the value and impact the app model will have on health care will increase as patients, providers, and administrators can more easily search, install, and ultimately be the market force that will drive innovation and value of EHR apps.

\section{Limitations}

We acknowledge several shortcomings of the current review. First, our review is limited only to apps currently available on the top 10 EHR app marketplaces by market share in the United States. We acknowledge there are other EHR vendors worldwide developing app marketplaces as well that were not reviewed here. During our review and research of EHR apps, we came across several other apps that claimed EHR integration that would have been included if they had been listed in an EHR marketplace. It would be unfeasible to know all apps that integrate with EHRs; nevertheless, we hope that as EHR marketplaces mature, these apps will become listed in the EHR marketplaces and organized in our review. Second, several apps had characteristics or features that could justify their classification under more than one category. In these cases, we endeavored to classify apps to the lowest level in the taxonomy as possible while still accurately reflecting the apps' primary purpose, which sometimes resulted in higher level classifications. In a few instances, when the information was insufficient to determine whether an offering was an app, we erred on the side of inclusion. As a result, a few apps in our taxonomy may have been inappropriately included. As further information becomes available, their inclusion and classification will be re-evaluated. Third, the review was conducted by two informaticists. We recognize that shortcomings, inaccuracies, and/or bias may exist in the interpretation and characterization of the apps. Independent input from a panel of expert stakeholders would increase robustness and validity of the EHR app review. Finally, the EHR app review represents a single point in time (February 2019). However, as new EHR apps get added to marketplaces and new app information becomes available, the results will become outdated. We anticipate conducting this review again in a few years to understand how EHR app marketplaces have evolved over time.

\section{Comparison With Prior Work}

The SMART app model was proposed in 2009 by Mandl et al [9]. Since then, EHR vendors have followed suit by building their own app marketplaces. We identified hundreds of apps in these marketplaces that allow integration with their respective EHR vendor. Current EHR marketplaces do not fully reflect the original proposal made by Mandl et al [9], which called for total substitutability of apps across any EHR by conforming to a single standard. Without conforming to a single standard as proposed by Mandl et al [9], each app would need to integrate with each EHR marketplace individually, as is the case today. For instance, we observed that 153 apps integrate with two or more EHRs. This does not quite meet the proposal made by Mandl et al [9] where an app lives on a single platform and can be integrated with any health system regardless of EHR vendor.

\section{Conclusions}

We characterized and organized a diverse and rich set of third-party EHR apps. This work provides an important reference for developers, researchers, and EHR customers to more easily search, review, and compare apps in EHR app marketplaces. While future research and validation among independent informaticists and stakeholders will increase the validity and value of this review, this work provides a strong foundation upon which future EHR app research will be established.

\section{Conflicts of Interest}

None declared.

\section{References}

1. Romano MJ, Stafford RS. Electronic health records and clinical decision support systems: impact on national ambulatory care quality. Arch Intern Med 2011 May 23;171(10):897-903 [FREE Full text] [doi: 10.1001/archinternmed.2010.527] [Medline: 21263077]

2. Adler-Milstein J, Jha AK. HITECH Act Drove Large Gains In Hospital Electronic Health Record Adoption. Health Aff (Millwood) 2017 Aug 01;36(8):1416-1422. [doi: 10.1377/hlthaff.2016.1651] [Medline: 28784734]

3. Samal L, Linder JA, Lipsitz SR, Hicks LS. Electronic health records, clinical decision support, and blood pressure control. Am J Manag Care 2011 Sep;17(9):626-632 [FREE Full text] [Medline: 21902448] 
4. Burke HB, Sessums LL, Hoang A, Becher DA, Fontelo P, Liu F, et al. Electronic health records improve clinical note quality. J Am Med Inform Assoc 2015 Jan;22(1):199-205 [FREE Full text] [doi: 10.1136/amiajnl-2014-002726] [Medline: 25342178]

5. Kalorama Information. EMR Market 2017: Electronic Medical Records in an Era of Disruption URL: https:/ /kaloramainformation.com/product/emr-market-2017-electronic-medical-records-in-an-era-of-disruption/ [accessed 2019-03-13]

6. Health IT Outcomes. EHR Failure: What's A Practice To Do? URL: https://www.healthitoutcomes.com/doc/ ehr-failure-what-s-a-practice-to-do-0001 [accessed 2019-03-12]

7. Healthcare IT News. 2012. 12 integration capabilities EHRs will need to have URL: https://www.healthcareitnews.com/ news/12-integration-capabilities-ehrs-will-need-have [accessed 2019-03-12]

8. Mandl K, Mandel J, Pfiffner P. Chapter 16 - An Apps-Based Information Economy in Healthcare. In: Sheikh A, Cresswell KM, Wright A, Bates DW, editors. Key Advances in Clinical Informatics. Cambridge, Massachusetts: Academic Press; 2017:227-236.

9. Mandl KD, Kohane IS. No small change for the health information economy. N Engl J Med 2009 Mar 26;360(13):1278-1281. [doi: 10.1056/NEJMp0900411] [Medline: 19321867 ]

10. Mandl KD, Kohane IS. Escaping the EHR trap--the future of health IT. N Engl J Med 2012 Jun 14;366(24):2240-2242. [doi: 10.1056/NEJMp1203102] [Medline: 22693995]

11. Mandl K, Kohane I, Christensen C, Chueh H, Frisse M, Kibbe D. smarthealthit.ork. 2009. Ten Principles for Fostering Development of an "iPhone-like" Platform for Healthcare Information Technology URL: http://smarthealthit.org/wp-content/ uploads/CHIP-HIT-Platform.pdf [accessed 2020-05-12]

12. Sittig DF, Wright A. What makes an EHR "open" or interoperable? J Am Med Inform Assoc 2015 Sep;22(5):1099-1101 [FREE Full text] [doi: 10.1093/jamia/ocv060] [Medline: 26078411]

13. smarthealthit.org. SMART App Gallery URL: https://gallery.smarthealthit.org/ [accessed 2019-03-12]

14. Mandel JC, Kreda DA, Mandl KD, Kohane IS, Ramoni RB. SMART on FHIR: a standards-based, interoperable apps platform for electronic health records. J Am Med Inform Assoc 2016 Feb 17 [FREE Full text] [doi: 10.1093/jamia/ocv189] [Medline: 26911829]

15. Mandl KD, Mandel JC, Kohane IS. Driving Innovation in Health Systems through an Apps-Based Information Economy. Cell Syst 2015 Jul;1(1):8-13 [FREE Full text] [doi: 10.1016/j.cels.2015.05.001] [Medline: 26339683]

16. healthit.gov. Connecting Health and Care for the Nation: A Shared Nationwide Interoperability Roadmap URL: https:/ /www.healthit.gov/sites/default/files/hie-interoperability/nationwide-interoperability-roadmap-final-version-1.0.pdf [accessed 2020-05-12]

17. Health IT Dashboard. Health Care Professional Health IT Developers URL: https://dashboard.healthit.gov/quickstats/pages/ FIG-Vendors-of-EHRs-to-Participating-Professionals.php [accessed 2019-03-12]

18. healthcareitnews.com. 2018. Allscripts buys Practice Fusion for $\$ 100$ million URL: https://www.healthcareitnews.com/ news/allscripts-buys-practice-fusion-100-million [accessed 2019-06-24]

19. Charmaz K. Constructing Grounded Theory: A Practical Guide Through Qualitative Analysis. New York City, NY: SAGE Publications; 2006.

20. Rasch R. The nature of taxonomy. Image J Nurs Sch 1987;19(3):147-149. [doi: 10.1111/j.1547-5069.1987.tb00613.x] [Medline: $\underline{3666771]}$

\section{Abbreviations}

API: application programming interface

CDS: clinical decision support

DME: durable medical equipment

EHR: electronic medical record

FHIR: Fast Healthcare Interoperability Resources

IT: information technology

ReST: Representational State Transfer

RIS/PACS: Radiology Information System/Picture Archiving and Communication System

SMART: Substitutable Medical Applications, Reusable Technologies 
Edited by C Lovis; submitted 08.11.19; peer-reviewed by K Mandl, C Fincham, M Kolotylo-Kulkarni, N Sakib; comments to author 09.02.20; revised version received 20.03.20; accepted 12.04.20; published 29.05.20

Please cite as:

Ritchie J, Welch B

Categorization of Third-Party Apps in Electronic Health Record App Marketplaces: Systematic Search and Analysis JMIR Med Inform 2020;8(5):e16980

URL: http://medinform.jmir.org/2020/5/e16980/

doi: $\underline{10.2196 / 16980}$

PMID: $\underline{32469324}$

CJordon Ritchie, Brandon Welch. Originally published in JMIR Medical Informatics (http://medinform.jmir.org), 29.05.2020. This is an open-access article distributed under the terms of the Creative Commons Attribution License (https://creativecommons.org/licenses/by/4.0/), which permits unrestricted use, distribution, and reproduction in any medium, provided the original work, first published in JMIR Medical Informatics, is properly cited. The complete bibliographic information, a link to the original publication on http://medinform.jmir.org/, as well as this copyright and license information must be included. 